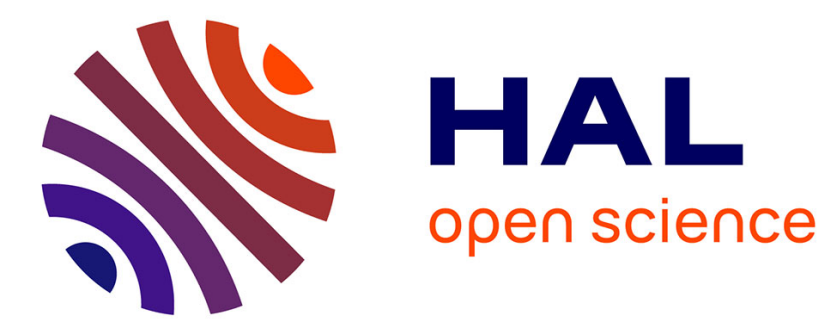

\title{
Les gueux sympathiques de Richard Brome dans A Jovial Crew; Or, the Merry Beggars (1641) \\ Pascale Drouet
}

\section{To cite this version:}

Pascale Drouet. Les gueux sympathiques de Richard Brome dans A Jovial Crew; Or, the Merry Beggars (1641). XVII-XVIII Revue de la Société d'études anglo-américaines des XVIIe et XVIIIe siècles , 2003, 56 (1), pp.105-120. 10.3406/xvii.2003.1820 . halshs-01840228

\section{HAL Id: halshs-01840228 \\ https://shs.hal.science/halshs-01840228}

Submitted on 16 Jul 2018

HAL is a multi-disciplinary open access archive for the deposit and dissemination of scientific research documents, whether they are published or not. The documents may come from teaching and research institutions in France or abroad, or from public or private research centers.
L'archive ouverte pluridisciplinaire HAL, est destinée au dépôt et à la diffusion de documents scientifiques de niveau recherche, publiés ou non, émanant des établissements d'enseignement et de recherche français ou étrangers, des laboratoires publics ou privés. 


\section{Les gueux sympathiques de Richard Brome dans A Jovial Crew;} Or, the Merry Beggars (1641)

\section{Pascale Drouet}

\section{Citer ce document / Cite this document :}

Drouet Pascale. Les gueux sympathiques de Richard Brome dans A Jovial Crew; Or, the Merry Beggars (1641). In: XVIIXVIII. Bulletin de la société d'études anglo-américaines des XVIle et XVIIle siècles. N56, 2003. pp. 105-120;

doi : https://doi.org/10.3406/xvii.2003.1820

https://www.persee.fr/doc/xvii_0291-3798_2003_num_56_1_1820

Fichier pdf généré le 27/03/2018 


\section{LES GUEUX SYMPATHIQUES DE RICHARD BROME DANS A JOVIAL CREW; OR, THE MERRY BEGGARS (1641)}

Au printemps $1641,{ }^{1}$ la dernière comédie de Richard Brome est jouée par "The King and Queen's Young Company," troupe également connue sous le nom de "Beeston Boys," au théâtre du Cockpit situé à Drury Lane. La date et le lieu sont significatifs à plus d'un titre. Le dramaturge présente $A$ Jovial Crew environ un an avant que le Parlement, où siège une majorité de puritains, ne donne l'ordre de fermer les théâtres. La fermeture officielle est ordonnée le 2 septembre 1642, mais les édifices ont été abandonnés plusieurs mois auparavant. ${ }^{2}$ Dans sa dédicace à Thomas Stanley, Richard Brome laisse entendre que sa pièce fut sans doute la dernière à être jouée au Cockpit: "it had the luck to tumble last of all in the epidemical ruin of the scene" (26-27). ${ }^{3}$ Le théâtre vit ses derniers jours.

Comme son titre l'indique, la comédie de Richard Brome propulse les mendiants et les miséreux sur le devant de la scène. Elle s'inscrit ainsi dans la lignée de trois pièces jacobéennes qui présentent des gueux sympathiques: Beggars Bush (1618) de John Fletcher et Philip Massinger, The Masque of Metamorphosed Gipsies (1621) de Ben Jonson, et The Spanish Gypsy (1623) de Thomas Middleton et William Rowley. L'originalité de Richard Brome est de mettre en scène une majorité de vrais gueux, et non les "beggars in disguise" de ses prédécesseurs, des gentilshommes exilés ou déchus qui revêtent des haillons pour contourner une forme de tyrannie. Or, sa comédie est jouée

\footnotetext{
1. "There are suggestions that the play was planned to open 25 April 1641, or thereabouts" (Gerald E. Bentley, The Jacobean and Caroline Stage, 7 vols. [Oxford: Clarendon, 1968] 3: 72).

2. "Before Parliament issued its closing order of September 1642, the King had deserted London, and the function of the Cockpit was gone" (Bentley 6: 283).

${ }^{3}$. Toutes les citations de la pièce et de son paratexte sont tirées de l'édition suivante: Richard Brome, A Jovial Crew; Or, The Merry Beggars, 1641, ed. Ann Haaker (London: Arnold, 1968).
} 
au Cockpit, théâtre privé aux prix prohibitifs pour les petites gens, ${ }^{4}$ elle s'adresse donc à un auditoire privilégié excluant tout élément marginal. En réalité, c'est le fait des circonstances et non d'un choix délibéré. Alfred Harbage précise: "Because of the growth of Puritanism and the gradual loss of the city audience, the theatres were depending more and more upon attendance by the gentry of Whitehall, the inns of court, and the environs of Holborn, Drury Lane, Fleet Street and the Strand." ${ }^{5} \mathrm{Vu}$ la conjoncture et les troubles politiques (les puritains condamnent le théâtre, le parlement démantèle le système de gouvernement autoritaire de Charles Ier et la guerre civile entre parlementaires et royalistes se profile), ${ }^{6}$ nous sommes en droit de supposer que cet auditoire est en proie à des inquiétudes diverses et à des pensées moroses. Le dramaturge annonce, d'ailleurs, dès le prologue: "jovial mirth is now grown out of fashion" (6). Le décalage social et "humoral" annoncé entre les joyeux traîne-semelles de la pièce et les spectateurs du Cockpit permet de prévoir un déplacement de sympathie de la scène vers la salle.

Pourtant, ce qui attire d'emblée l'attention dans A Jovial Crew, c'est le traitement sympathique de la gueuserie. La sympathie, dans ce cas précis, doit se définir a contrario: contrairement à certaines représentations de la fin du XVIe siècle, les gueux ne sont pas des figures antipathiques provoquant un sentiment de rejet, de dégoût, de peur ou de culpabilité. Non seulement les aspérités de la mendicité et de la maladie ont été gommées, mais les signes mêmes de la pauvreté ont été inversés. Les joyeux drilles deviennent sympathiques au sens où ils n'incarnent plus le visage inquiétant de l'altérité. La bienveillance que ressent le gentilhomme Oldrents pour les mendiants déguenillés qu'il abrite et la philanthropie dont il fait preuve à leur égard semblent alors aller de soi. Ne nous y trompons pas cependant: ses actes charitables ne sont pas complètement désintéressés; ils pourraient relever davantage de la contagion affective que de la sympathie morale. En effet, les traînesavates enjoués sont pris dans une économie du divertissement visant à soigner la mélancolie du vieil Oldrents. Autrement dit, nous pouvons nous demander si les gueux ne sont pas présentés comme sympathiques parce qu'ils participent à un enjeu thérapeutique à double niveau (pour les autres personnages de la comédie et pour les spectateurs du Cockpit), et

${ }^{4}$. Voir Andrew Gurr, Playgoing in Shakespeare's London (Cambridge: Cambridge UP, 2000) 175-82.

5. Alfred Harbage, Cavalier Drama: an Historical and Critical Supplement to the Study of the Elizabethan and Restoration Stage (London: Oxford UP, 1936) 149.

6 . Voir François-Joseph Ruggiu, L'Angleterre des Tudors aux premiers Stuarts, 15091660 (Paris: Sedes, 1998) 52-59. 
si le dramaturge ne détourne pas le pittoresque sociologique au profit d'une sympathie de classe.

\section{La représentation sympathique des gueux: l'inversion des signes de la misère}

Les gueux bonhomme de Richard Brome sont très loin des "sturdy beggars," ces imposteurs oisifs, robustes mais retors, parfois menaçants, que dépeint minutieusement Thomas Harman, en 1566, dans sa typologie de mendiants, ${ }^{7}$ et que les "Poor Laws" élisabéthaines condamnent avec une sévérité et un acharnement qui trahissent alors une véritable phobie. ${ }^{8}$ Les six proclamations contre les vagabonds, qui ponctuent le règne de Charles Ier de 1628 à 1634, s'inscrivent dans la lignée élisabéthaine et attestent que l'aversion envers les "sturdy beggars" est toujours vivace. ${ }^{9}$

7. Voir Thomas Harman, A Caveat or Warning for Common Cursitors, Vulgarly Called Vagabonds, 1566, The Elizabethan Underworld: a Collection of Tudor and Early Stuart Tracts and Ballads Telling of the Lives and Misdoings of Vagabonds, Thieves, Rogues and Cozeners, and Giving some Account of the Operation of the Criminal Law, ed. Arthur V. Judges (1930; London: Routledge, 1965) 61-118.

${ }^{8}$. Voir en particulier "An Acte for the punishment of vacabondes, and for the releif of the poore and impotent" (14 Eliz., c. 5), 1572, et "An Acte for the punyshment of rogues, vagabondes and sturdy beggars " (39 Eliz., c. 4), 1597, dans Richard H. Tawney et Eileen Power, eds., Tudor Economic Documents, 3 vols. (London: Longman, 1924) 2: 328-331 et 2: 346-354.

9. Il s'agit, dans l'ordre chronologique, de: "A Proclamation for the execution of the Statutes made against Rogues and Vagabonds" (Whitehall, 16 February 1628); "A Proclamation for the speedy sending away of the Irish Beggers out of this Kingdome, into their owne Countrey, and for the suppressing and ordering of the English Rogues and Vagabonds, according to the Lawes" (Greenwich, 17 May 1629); "A Proclamation commanding the due execution of the Lawes made for setting the poore on worke" (Greenwich, 17 May 1629); "A Proclamation for quickening the Lawes made for the reliefe of the poore, and the suppressing, punishing, and setling of the Sturdy Rogues, and Vagabonds" (Whitehall, 23 April 1630); "A further Proclamation for the suppressing and punishing of Rogues and Vagabonds, and Reliefe of the Poore, according to the Law" (Theobalds, 17 September 1630); "A Proclamation for the speedie sending away of the Irish Beggars out of this Kingdome into their owne Countrey, and for the suppressing of English Rogues and Vagabonds, according to Our Lawes" (Whitehall, 12 February 1634). La proclamation du 23 avril 1630 s'ouvre en précisant qu'elle reprend effectivement les Poor Laws élisabéthaines: "Whereas many excellent Lawes and Statutes with great judgement and providence have been made in the times of Our late deare and royall Father, and of the late Queene Elizabeth, for the reliefe of the impotent, and indigent poore, and for the punishing, suppressing, and setling of the sturdy Rogues, and Vagabonds, which Lawes and Statutes, if they were duely observed would be of exceeding great use for the peace and plentie of this Realme, but the neglect thereof is the occasion of much disorder, and many insufferable abuses" (James P. Larkin, ed., Stuart Royal Proclamations, Vol. 2: Royal Proclamations of King Charles 1, 1625-1646 [Oxford: Clarendon, 1983] 262). 
Chez le dramaturge caroléen en revanche, ils ne donnent pas lieu à une condamnation morale; la mendicité n'est plus présentée comme un vice qu'il faut éradiquer chez les "mauvais pauvres," 10 mais comme un besoin vital, comme une compulsion naturelle, ce que le personnage de Springlove appelle: "this predominant sway / Of nature" (1.1.242-43). Les pauvres hères de Richard Brome font l'objet d'une projection utopique dans un "green world"11 idéalisé avec, d'une part, des renversements thématiques quasi antonymiques et, d'autre part, une inversion poétique propre au mode héroï-comique, ou encore à ce que Charles Perrault appelle "le burlesque retourné."12

Les gais lurons (et larrons) qui vivent de la charité sans cesse renouvelée du vieil Oldrents ne présentent aucun des grands traits propres aux miséreux: la faim a été remplacée par le festin, l'inquiétude s'est transformée en joie de vivre, l'isolement a laissé place à la communauté, l'exclusion s'est changée en hospitalité. Ces inversions sont d'autant plus perceptibles que le théâtre de Shakespeare, toujours joué sous l'Angleterre de Charles Ier, dans les théâtres publics et privés, ${ }^{13}$ fournit des contre-exemples qui servent de repoussoirs aux traîne-savates insouciants de Richard Brome.

Dans A Jovial Crew, le principe de réalité (l'errance et la faim) est relayé d'emblée par le principe de plaisir (l'hébergement et le festin). Les gueux sont gracieusement logés dans la grange du charitable Oldrents transformée en "guesthouse" (1.1.261) où l'intendant dépose quotidiennement "the breadbasket, the beef kettle, and the beer bumbards" (1.1.262-63). Or, dans le deuxième acte, l'utopie de la table d'hôte est relayée par l'utopie de la corne d'abondance: on nous montre les

${ }^{10}$. La distinction entre les "bons pauvres" (les pauvres physiquement diminués ayant droit d'aumône) et les "mauvais pauvres" (les imposteurs oisifs qui vivent de la charité au lieu de travailler) est établie par Henri Sauval dans Histoire et recherche des antiquités de la ville de Paris, 3 vols. (Paris: Jacques Chardon, 1724) 1: 510.

${ }^{11}$. L'expression est de Northrop Frye dans "The Argument of Comedy," Shakespeare, Modern Essays in Criticism, ed. Leonard Fellow Dean (New York: Oxford UP, 1967) 85.

${ }^{12}$. Dans Parallèle des anciens et des modernes (Paris, 1692), Charles Perrault définit "le burlesque retourné" comme traitant "des choses les plus communes et les plus abjectes en des termes pompeux et magnifiques." Cité par Gérard Genette, Palimpsestes. La littérature au second degré (Paris: Seuil, 1982) 185.

13. "But the fashionable theatres, too - the Blackfriars, the Phoenix, and the Salisbury Court - indulged this Elizabethan nostalgia by including many old 'popular' plays in their repertories" (James Bulman, "Caroline Drama," The Cambridge Companion to English Renaissance Drama, ed. Albert Richard Braunmuller et Michael Hattaway [Cambridge: Cambridge UP, 1999] 353). Voir également Anne Barton, "Harking Back to Elizabeth: Ben Jonson and Caroline Nostalgia," A Journal of English Literary History 48 (1981): 706-31. 
pauvres déguenillés en train de ripailler joyeusement et d'entonner, dans le jargon des colporteurs, une chanson énumérant toutes les victuailles (bacon, pain, beurre, fromage, potage, porc, poulet) dont ils se repaissent. Richard Brome met ici en scène ce que Stephen Greenblatt appelle "a dream of superabondance"; ${ }^{14}$ ses traîne-misère sont aux antipodes des crève-la-faim efflanqués, des épouvantails pathétiques que recrute tant bien que mal Falstaff dans 1 Henry $I V$, et pour lesquels la corne d'abondance ne délivre qu'épluchures et eaux grasses. ${ }^{15}$

Selon un procédé similaire, c'est la joie de vivre, la solidarité communautaire et l'insouciante liberté dont jouissent les gueux qui sont mises en relief par le dramaturge. Les boute-en-train en haillons sont les premiers chantres de leur bien-être:

From hunger and cold, who lives more free,

Or who more richly clad than we?

Our bellies are full; our flesh is warm;

And, against pride, our rags are a charm.

Enough is our feast, and for tomorrow

Let rich men care; we feel no sorrow. (1.1.339-44)

Loin de se sentir isolés et exclus, ils ont recréé une microsociété dont la devise pourrait être: "liberté, communauté, gaieté." Paradoxalement, leur sort n'est plus pitoyable mais enviable, car ils apparaissent libérés des contraintes de la société, des devoirs civiques, des peurs liées aux troubles politiques. Vincent, l'un des gentilshommes, déclare: "With them there is no grievance or perplexity; / No fear of war, or state disturbances. / No alteration in a commonwealth, / Or innovation, shakes a thought of theirs" (4.2.90-93). Ce qui peut se lire comme un fantasme d'inversion culmine avec cet éloge du mode de vie des va-nu-pieds: la dépendance prosaïque qui (dé)structure en réalité leur quotidien s'efface pour laisser place à une liberté a contrario qui s'apparente à une construction intellectuelle.

Cette présentation utopique de la gueuserie, ce que Rosemary Gaby appelle "a romanticized version of vagabond society,"16 annule tout sentiment de sympathie dans son acception étymologique: ${ }^{17}$ comment partager les souffrances des pauvres hères, comment compatir puisqu'ils

\footnotetext{
${ }^{14}$. Stephen Greenblatt, Shakespearean Negotiations: The Circulation of Social Energy in Renaissance England (1987; Oxford: Clarendon, 1990) 41.

${ }^{15}$. "I had a hundred and fifty tattered prodigals lately come from swine-keeping, from eating draff and husks. A mad fellow met me on the way, and told me I had unloaded all the gibbets and pressed the dead bodies. No eye had seen such scarecrows" (4.2.34-38).

${ }^{16}$. Rosemary Gaby, "Of Vagabonds and Commonwealths: Beggars' Bush, A Jovial Crew, and The Sisters," Studies in English Literature, 1500-1900 34 (1994): 408.
} 
semblent mener une vie joyeuse et digne d'envie? Dans A Jovial Crew, l'expérience et l'expression de la souffrance sont oblitérées, et nul sentiment de compassion ne peut donner lieu à une réflexion sur les inégalités et les injustices sociales. En fait, la réécriture antonymique à laquelle recourt Richard Brome pourrait répondre à une stratégie qui serait non de sensibiliser, mais de déculpabiliser l'auditoire. Car l'inversion apparaît sur le mode non seulement thématique mais aussi poétique: le traitement héroï-comique de certaines scènes confirme la stratégie de déculpabilisation et, même, l'inclut dans une économie du divertissement. C'est le comble du renversement puisque ce qui devait apitoyer vient à distraire. Les gueux de Richard Brome sont sympathiques dans la mesure où ils ne crient pas haut et fort leur misère; ils le deviennent bien davantage lorsqu'ils se révèlent distrayants.

Au fil de sa comédie, le dramaturge présente plusieurs tableaux ayant pour sujet les célébrations bon enfant des traine-savates. Au quatrième acte, le dernier tableau nous montre les festivités nuptiales, entre une aveugle et un vieil estropié, qui s'accompagnent d'un poème héroïcomique: l'épithalame du gueux-poète compare les invalides en haillons à des elfes féeriques et convoque à leur intention dieux et déesses mythologiques. ${ }^{18}$ Le recours au "burlesque retourné" est à la source du divertissement pour les personnages-spectateurs et, surtout, pour l'auditoire du Cockpit. À ses yeux, il est fort possible que la danse grotesque des traîne-misère, exécutée à l'aveuglette et clopin-clopant, ait pris des allures d'antimasque. Les mendiants festifs peuvent alors être perçus dans leur altérité, avec ce qu'elle comporte de rusticité et de grossièreté, parce qu'ils sont pris dans une économie pittoresque et récréative. Le décalage stylistique, la "disconvenance"19 entre le sujet (vulgaire) et son traitement (noble), permet sans danger de montrer l'Autre dans sa différence puisqu'il est circonscrit dans un genre littéraire (le poème héroï-comique) ou dans un spectacle (l'antimasque) codés qui font que seules ses qualités divertissantes seront retenues.

Par des effets presque opposés, les inversions thématiques et poétiques contribuent donc à la représentation sympathique des mendiants. Les premières, en mettant en avant les avantages utopiques de

\footnotetext{
${ }^{17}$. Voir Alain Rey, Dictionnaire historique de la langue française, 3 vols. (Paris: Dictionnaires Le Robert, 1998) 3: 3720-21 (art. "sympathie").

${ }^{18}$. L'épithalame commence en ces termes: "To the blind virgin of fourscore, / And the lame bachelor of more, / How Cupid gave her eyes to see, / And Vulcan lent him legs; How Venus caus'd their sport to be / Prepar'd with butter'd eggs" (4.2.66-71).

${ }^{19}$. Le terme est de Charles Perrault dans Parallèle des anciens et des modernes. Cité dans Genette 185.
} 
la gueuserie, ramènent l'Autre au Même; les secondes soulignent, certes, les différences de l'Autre mais en les définissant comme pittoresques et distrayantes. Incarnation paradoxale de la joie de vivre et du rire, les vanu-pieds de Richard Brome ne sont ni fuis ni montrés du doigt, mais recherchés pour leurs talents d'amuseurs. La bienveillance, la charité et l'hospitalité qui s'exercent à leur égard risquent donc de s'en trouver biaisées.

\section{La contagion affective comme remède à la mélancolie}

Dès la première scène de $A$ Jovial Crew, Oldrents est présenté comme un gentilhomme qui se montre charitable envers les traîne-misère et qui a l'affection de son entourage proche et lointain, fortuné ou non, comme le résume la question rhétorique de son ami Hearty: "Have you not all the praises of the rich, / And prayers of the poor?" (1.1.68-69). Son comptable Springlove termine l'énumération récapitulative de ses dépenses en soulignant sa générosité: "And lastly, not the least to be remember'd, / Your large benevolence to the poor" (1.1.137-38). Pourtant, aux yeux de son serviteur Randall, cette charité est mal ciblée; elle entretient l'oisiveté de ses bénéficiaires:

It might be better though (if old Randall, whom you allow to talk, might counsel) to help to breed up poor men's children, or decayed laborers, past their work, or travail; or towards the setting of the poor, young married couples, than to bestow an hundred pound a year . . . to maintain in begging such wanderers as these, that never are out of their way; that cannot give account from whence they came or whither they would; nor of any beginning they had, or any end they seek, but still to stroll and beg till their bellies be full, and then sleep till they be hungry. (1.1.27586)

Or, les mendiants que secourt Oldrents ne lui sont pas si antipathiques qu'il le prétend; en réalité, ils le divertissent. Rendall admet, en effet, peu après: "they know I love 'em well enough. I have had merry bouts with some of 'em. . . . They are indeed my pastime" (1.1.299-302). Sa critique peut donc se (re)lire comme une parodie du discours officiel qui, depuis les Poor Laws élisabéthaines, condamne le désœuvrement, prône une charité non plus individuelle mais étatisée, et prétend corriger les mauvais pauvres en les enfermant dans des maisons de correction appelées "bridewells." Car dans l'Angleterre caroléenne, "la grande proscription de l'oisiveté"20 reste d'actualité, d'autant que la récession

\footnotetext{
${ }^{20}$. L'expression est de Michel Foucault dans Histoire de la folie à l'âge classique (Paris: Gallimard, 1972) 102.
} 
augmente le nombre de pauvres. L'autre solution que présente Richard Brome en ces temps troublés, "these sad and tragic days" (3) comme il l'annonce dans son prologue, c'est de considérer les traine-savates comme des instruments festifs, propices à un regain de bonne humeur et de joie de vivre. Ce qui signifie que la charité exercée à leur égard n'est pas entièrement désintéressée.

Dans A Jovial Crew, la sympathie morale qu'éprouvent Oldrents, Springlove et Hearty envers les joyeux drilles a effectivement ses limites. L'hospitalité ne s'étend ni au questionnement ni à la compréhension de la misère. Lorsque le prêtre des gueux commence à dévoiler l'envers du décor, Hearty le réinscrit dans une économie du divertissement dont il n'aurait pas dû sortir: "you are too grave. Let us hear and see something of your merry grigs, that can sing, play gambols, and do feats" (2.2.22830). Gagné par la mélancolie, Oldrents ne porte plus à ses hôtes en guenilles qu'un intérêt qui dissimule à peine un processus d'instrumentalisation. Il projette de doubler leur aumône pour qu'ils viennent, en plus grand nombre, remplir sa demeure de chants festifs, de ripailles divertissantes au sens pascalien du terme. Sa charité s'affiche alors clairement comme un moyen visant à lui faire oublier son humeur mélancolique: "I mean to send forty miles' circuit at the least / To draw in all the beggars can be found, / And such devices we will have for jollity / As fame shall boast it to all posterity" (2.2.136-40). ${ }^{21}$ Ce que recherche le personnage de Richard Brome, c'est une forme déviante de la sympathie que Max Scheler appelle la contagion affective. Dans Nature et formes de la sympathie, le philosophe allemand la définit ainsi:

[I]1 n'y a là [dans la contagion affective] ni intention affective à l'égard de la joie ou de la souffrance d'autrui, ni participation à ses expériences externes. . . . La contagion affective peut être à nouveau mise au service de la volonté consciente. C'est ce qui arrive lorsque nous cherchons une "distraction," lorsque, sans être, précisément, dans une "joyeuse disposition," nous nous rendons dans une joyeuse "Société" ou dans une fête "pour tuer le cafard," dans l'"espoir de subir la contagion de la gaité; d'être entraîné dans le courant de la gaîté générale." Il est évident que lorsque nous disons que "nous serions heureux de voir autour de nous des visages gais," nous cherchons, non à partager (par sympathie) la joie des autres, mais uniquement notre propre plaisir que nous attendons précisément de la contagion. ${ }^{22}$

\footnotetext{
${ }^{21}$. C'est nous qui soulignons.

22. Max Scheler, Nature et formes de la sympathie, trad. Maurice Lefebvre (Paris: Payot, 1928) 30-32. Max Scheler (1874-1928) a consacré une importante partie de son œuvre à la phénoménologie de l'affectivité.
} 
Or, n'est-ce pas justement une forme de contagion affective, plus égoïste qu'altruiste, que Robert Burton, en 1621, recommande au mélancolique dans The Anatomy of Melancholy? Dans la rubrique intitulée "Mirth and merry Company, faire Objects, Remedies," il prescrit en effet: "Feast often, and use friends not still so sad, / Whose jests and merriments may make thee glad." ${ }^{23}$ Puis il conclut en insistant sur la vertu thérapeutique de l'entreprise: "merry company is the only medicine against melancholy." ${ }^{24}$ Le personnage de Richard Brome suit la prescription à la lettre; dans la compagnie des traîne-semelles enjoués, il cherche un divertissement certes, mais qui soit aussi une remède radical: "I'll purge my house of stupid melancholy" (2.2.119), déclare-t-il résolument à son ami Hearty. Les danses et chants de la joyeuse équipe parmi laquelle figurent des musiciens déchus, chassés de Londres et réduits à la mendicité pour avoir entonné des airs diffamatoires, ${ }^{25}$ apparaissent véritablement comme des "pills to purge melancholy," expression qui sera consacrée par John Playford en 1698, avec son recueil de chansons gaies et égrillardes: Wit and Mirt; Or, Pills to purge melancholy. ${ }^{26}$

La représentation sympathique des gueux, où les signes de la misère sont inversés, pourrait donc participer d'une économie curative à deux niveaux, les patients étant, sur la scène du Cockpit, le personnage mélancolique d'Oldrents et, dans la salle, le public lui-même. Car les festivités des gueux ne sont-elles pas à Oldrents ce que la comédie de Richard Brome est aux spectateurs du Cockpit? Dans son introduction, Ann Haaker nous renseigne, en effet, sur l'attente de ces derniers: "on the eve of the civil war melancholy had replaced jovial mirth 'now grown out of fashion'. Theater audiences demanded escapism from the sordid realities." ${ }^{27}$ La mélancolie généralisée à laquelle il est fait référence est probablement l'expression d'une nostalgie pour la "Merry Old England," cette Angleterre calendaire et festive qui ne pliait pas encore sous le joug puritain. Quant à l'idée d'une thérapie effectuée par l'intermédiaire du

${ }^{23}$. Robert Burton, The Anatomy of Melancholy, 1621, ed. Thomas C. Faulkner, Nicolas K. Kiessling et Rhonda L. Blair, 6 vols. (Oxford: Clarendon, 1990) 2: 121. C'est nous qui soulignons.

${ }^{24}$. Burton 2: 123 .

25. "We have musicians, too, among us: true merry beggars indeed, that being within the reach of the lash for singing libelous songs at London, were fain to fly into our covey, and here sing all our poet's ditties. They can sing anything most tenably, sir, but Psalms" $(1.1 .433-37)$

${ }^{26}$. John Playford (1623-1686), éditeur londonien spécialisé dans l'édition musicale et notamment connu pour son traité The English Dancing Master; Or, Plaine and Easie Rules for the Dancing of Country Dances (1651).

27 . Brome xv (introduction). 
théâtre, Richard Brome l'a déjà mise au centre de la comédie qu'il a fait jouer en 1638, The Antipodes, en usant d'un procédé de mise en abyme. ${ }^{28}$ Dans A Jovial Crew, l'effet n'est plus centripète mais centrifuge: l'enjeu thérapeutique dépasse les limites de la représentation. Dans l'éloge versifié qu'il fait de $A$ Jovial Crew, Alexander Brome écrit, s'adressant au dramaturge en personne: "Thou'rt th' age doctor now, for since all go / To make us poor, thou mak'st us merry too." 29 Pour filer la métaphore médicale, disons que Richard Brome saupoudre de l'illusion théâtrale, pour arrêter l'hémorragie mélancolique, comme s'il s'agissait d'une poudre de sympathie. ${ }^{30}$

Nous pourrions donc penser que le dramaturge témoigne indirectement d'une sympathie de classe, au sens où son attention semble se porter, en fin de compte, non sur des traîne-misère pris dans une réalité sociale, mais sur un gentilhomme en proie à une humeur mélancolique (c'est-à-dire, aristocratique) et, par effet de miroir, sur un auditoire privilégié dont il faut soigner l'esprit morose. Or, Richard Brome prend soin de réinscrire ses joyeux va-nu-pieds, ceux qu'il appelle, dans sa dédicace à Thomas Stanley, "these harmless beggars" (32), dans une économie d'échange.

\section{Les gueux dans une économie d'échange: vers une société en sympathie?}

La contagion affective (au contact des gueux ou des acteurs) comme remède à la mélancolie est à replacer dans une économie d'échange, ce que Stephen Greenblatt appelle "a circulation of social energy," 31 où se lisent en filigrane des festivités marginales l'expression de la reconnaissance, la dialectique divertir/subvertir et une tentative d'inclusion.

La joyeuse équipe qui festoie quotidiennement aux frais d'Oldrents propose elle-même sa joie de vivre comme une monnaie d'échange, ou plutôt comme une marque de gratitude. À l'arrivée de Springlove, l'un des

${ }^{28}$. Voir Athéna Efstathiou-Lavabre, "Le Théâtre dans le théâtre: Permanence d'une structure baroque dans The Antipodes (1638) de Richard Brome," BSÉAA XVII-XVIII 54 (2002): 27-43.

29. "To Master Richard Brome, upon his Comedy Called A Jovial Crew; Or, The Merry Beggars" (33-34). L'intégralité du poème se trouve dans Brome 13.

30. "Poudre de sympathie désignait (1643) un remède à la mode au XVIIe siècle, formé de vitriol calciné que l'on faisait agir sur du sang prélevé à un blessé pour arrêter l'hémorragie" (Rey 3: 3720).

31. Greenblatt 1-20. 
boute-en-train s'improvise "Master of the Revels" et dit avec enthousiasme:

Shall we dance, shall we sing, to welcome our king?

Strike up pipers a merry, merry dance

That we on our stampers may foot it and prance,

To make his heart merry as he has made ours,

As lustic and frolic as lords in their bowers. (1.1.365-69).

Il conclut, après la danse enlevée des gueux: "we have not that rag among us that we will not dance off to do you service, we being all and only your servants, most noble sir" (1.1.371-73). Or, dans sa dédicace à Thomas Stanley, connu pour ses activités de mécène dans le monde des lettres, Richard Brome présente sa comédie dans des termes similaires: "I present this as a testimonial of my gratitude or recompense for your favors" (14-15). Comme les gueux déguenillés qu'il met en scène, il offre à son bienfaiteur de la gaieté en partage: "since the times conspire to make us all beggars, let us make ourselves merry; which (if I am not mistaken) this drives at" (30-32).

Le parallèle entre Thomas Stanley et le charitable Oldrents est, en fait, annoncé indirectement par le dramaturge: "we all know beggars use to flock to great men's gates" (9-10), qui file ensuite la métaphore de la mendicité. Il fait clairement allusion à l'agonie du théâtre, et c'est à sa comédie même qu'il applique l'image de l'indigent en quête d'oboles: "All the arguments I can use to induce you to take notice of this thing of nothing is that it had the luck to tumble last of all in the epidemical ruin of the scene, and now limps hither with a wooden leg to beg an alms at your hand" (25-29). Richard Brome sollicite la protection matérielle et la caution morale de Thomas Stanley en lui demandant d'abriter ses traînemisère inoffensifs auxquels il dit s'agréger en reprenant leur refrain: "Duly and truly pray for you" (35). Alors que le prologue se définit généralement comme "un encadrement déréalisant la fiction théâtrale," ${ }^{32}$ c'est le procédé inverse qui est mis en œuvre dans l'épître dédicatoire: l'illusion semble calquer le réel. Selon un schéma plus attendu, ${ }^{33}$ la métaphore de la mendicité est reprise dans l'épilogue, qualifié à juste titre par Springlove de "begging Epilogue" (5.1.501). Il s'ouvre ainsi: "Tho' we are, now, no beggars of the crew, / we count it not a shame to beg of you" (5.1.503-04). C'est la face humble de l'économie d'échange entre le dramaturge, son mécène et son public. Or, cette métaphore n'a pas qu'une

\footnotetext{
32. Patrice Pavis, Dictionnaire du Théâtre (Paris: Dunod, 1996) 273 (art. "prologue").

${ }^{33}$. Ce sont John Fletcher et Philip Massinger qui, les premiers, juxtaposent le niveau littéral et le niveau métaphorique du verbe "to beg" en confiant l'épilogue aux mendiants dans Beggars Bush (1618).
} 
valeur rhétorique: elle peut également se lire comme une stratégie idéologique de nivellement social et, donc, de non-exclusion des éléments marginaux. Le fait que le dramaturge recourt aux gueux et à leur mode de vie sur le plan dramatique (les personnages) et sur le plan poétique (la métaphore), invite à une lecture réaliste certes (à la veille de la guerre civile, l'Angleterre de Charles Ier est gangrenée par la pauvreté), mais aussi à une lecture symbolique: l'intégration du traîne-savates dans l'échiquier social, c'est-à-dire son insertion dans une économie d'échange.

En proposant leur joie de vivre comme monnaie d'échange, comme remède à la mélancolie, les gueux de Richard Brome illustrent ce que James Bulman appelle "a popular theory of comic catharsis." ${ }^{34}$ Or, s'ils amusent les spectateurs (parfois à leurs dépens), ils les amènent aussi à rire d'eux-mêmes; ils sont au cœur d'une économie d'échange dans la mesure où ils ont un rôle pivot: ils divertissent autant qu'ils subvertissent. Deux exemples s'offrent à nous.

Le premier acte de A Jovial Crew s'achève sur l'hymne au printemps qu'entonnent gaiement les insouciants va-nu-pieds. Leur chant commence ainsi:

Come, come; away: the spring

(By every bird that can but sing,

Or chirp a note) doth now invite

Us forth, to taste of his delight.

In field, in grove, on hill, in dale;

But above all the nightingale. (2.1.473-78). (Italiques dans le texte; souligné par moi)

Il pourrait s'entendre comme un pastiche de "Come again: Sweet Love doth now invite," air du musicien John Dowland, publié dans The First Book of Ayres en 1597. Il présente particulièrement des analogies avec le premier couplet et le refrain de "Come again: Sweet Love doth now invite," qui ont pour paroles:

Come again, sweet love doth now invite

Thy graces that refrain

To do me due delight,

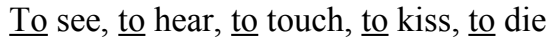

With thee again in sweetest sympathy. ${ }^{35}$

\footnotetext{
34. Bulman 368 .
}

35. John Dowland (1563-1626), The First Book of Ayres, 1597, ed. Edmund H. (London: Stainer and Bell, 1965). C'est nous qui soulignons les similitudes dans les deux citations. Les italiques de la première citation sont celles du dramaturge qui indique ainsi qu'il s'agit d'un chant. 
Ce qui est moqué dans le contexte de la comédie de Richard Brome, ce n'est pas tant l'idée d'une harmonie amoureuse, de deux corps résonnant en sympathie, que la figure du mélancolique incarnée par John Dowland - le ténébreux musicien aurait, peut-être, inspiré à Shakespeare le personnage de Hamlet. L'idée d'une sympathie d'humeur (et de classe) pour le mélancolique est donc minée par le pastiche que le dramaturge prête à ses traîne-semelles; Richard Brome réintroduit donc, avec subtilité, la notion de juste mesure, la "mètrios" chère aux Grecs, et s'inscrit dans le sillage shakespearien en se servant des personnages marginaux pour donner à ses spectateurs une vision relativiste de la société et de son temps.

Le second exemple de divertissement subversif se rencontre, à la fin du quatrième acte, sous la forme d'un projet de masque, pour célébrer le mariage des invalides, avec les gueux eux-mêmes comme acteurs. Ce que le poète-gueux présente comme "a masque or a comedy presented tonight, in honor of the old couple" (4.2.172-73), ce que Rosemary Gaby appelle "a vagabond pageant," 36 est en réalité une parodie de masque où sont tournées en dérision les rivalités entre la cour, la ville et la campagne, où le théâtre des vanités est ridiculisé: l'ensemble des personnages allégoriques "the country," "the city," "the court," "Divinity," "Law" finit à "Beggars' Hall" (4.2.217), lieu symbolique de la chute sociale et du marasme économique. La parodie est à son comble lorsque le poète-gueux conclut en précisant que le masque sera en l'honneur de la confrérie des traîne-savates, "for the honor of our calling" (4.2.215-16): leur masque, non conforme à la tradition, ne prend pas le monarque comme dédicataire exclusif et comme centre de perspective, mais est à la gloire de la communauté mendiante. Cette parodie rappelle aux spectateurs que les deux pôles de la hiérarchie sociale, le roi et le gueux, doivent être mis en perspective pour avoir un point de vue relativiste.

Malgré une époque troublée qui voit la montée des animosités politiques, Richard Brome ne tente-t-il pas, au moyen de sa comédie, de rappeler que la société vers laquelle il faudrait tendre est une société dont les différentes composantes sont en sympathie, c'est-à-dire, pour expliciter la métaphore musicale, qu'elles résonnent les unes par rapport aux autres, les cordes des unes faisant vibrer celles des autres? Dans $A$ Jovial Crew, le microcosme social n'est plus pris dans une perspective pyramidale mais plutôt organique, propice à la mise en relation, à l'échange concerté entre ses divers éléments. S'entendent alors par résonance harmonique, par sympathie au sens musical du terme, le

\footnotetext{
${ }^{36}$. Gaby 412.
} 
gentilhomme mélancolique et le boute-en-train loqueteux, l'acteur et le spectateur, le dramaturge et le mécène, l'illusion théâtrale et la réalité.

Par l'idée d'une société en sympathie ne condamnant et n'excluant ni les va-nu-pieds ni les acteurs, mais les inscrivant, au contraire, dans une économie d'échange et reconnaissant leurs qualités divertissantes et leur valeur curative, Richard Brome fait indirectement une apologie du théâtre, apologie qui, en 1641, s'apparente à un plaidoyer. En opérant cet ultime transfert de sympathie et en exprimant une sympathie d'ordre idéologique, il apparaît comme représentatif des dramaturges caroléens dont James Bulman nous dit:

Throughout the Caroline period, ... the dramatists were intensely preoccupied with the ideological implications of theatre. Threats of court censorship, a shrinking of audiences, and a growing opposition to the theatre among more zealous puritans made dramatists sensible of the precariousness of a livelihood they had once taken for granted; and in their plays, consequently, theatrical self-reference took a decidedly introspective turn. ${ }^{37}$

En soulignant la visée thérapeutique de l'illusion théâtrale, Richard Brome va à l'encontre du stéréotype puritain qui associe au théâtre non la guérison mais la contagion: contagion littérale en cas de peste, contamination morale le reste du temps puisque les représentations, selon les termes de Phillip Stubbes dans The Anatomie of Abuses, "norish ydleness." ${ }^{38}$ Cette accusation, qui fait du théâtre le réceptacle du vice, ne date pas de l'époque caroléenne mais remonte aux années 1570 et 1580, périodes des premiers écrits de détracteurs puritains tels que John Northbrook, Stephen Gosson et Phillip Stubbes; ${ }^{39}$ ils sont relayés, sous le règne de Charles Ier, par William Prynne qui publie, en 1633, son volumineux Histriomastix.

Or, il est intéressant de rappeler que, sous le règne d'Élisabeth Ière comme sous celui de Charles Ier, les maux dont on accuse les acteurs dans les pamphlets contre le théâtre ont des traits communs avec les justifications qu'avancent les proclamations royales pour châtier les "sturdy beggars": la mise en scène du corps à but lucratif, ou l'art de contrefaire, l'oisiveté et le mauvais exemple qu'elle véhicule, alimentent

37. Bulman 368 .

${ }^{38}$. Phillip Stubbes, The Anatomie of Abuses, 1583, Phillip Stubbes's Anatomy of Abuses in England in Shakespeare's Youth, A.D. 1583, ed. Frederick J. Furnivall. (London: Trübner, 1877) 144.

39. Voir Jonas Barish, The Antitheatrical Prejudice (Berkeley: U of California P, 1981). 
la mauvaise presse qu'ils ont de part et d'autre. Dans A Jovial Crew, Richard Brome fait allusion à l'amalgame qui s'installe entre baladins et traîne-semelles: le personnage de Justice Clark, incarnant la loi et l'autorité, confond "beggars," "vagabonds" et "strolling players" (5.1.25153). Le sursis que le juge de paix est prêt à leur accorder dépend du bon plaisir d'Oldrents, ainsi qu'il le souligne: "They are upon purgation. If they can but present anything to please you, they may escape the law" (5.1.253-54). Sont désignés, dans le cadre de la comédie, les joyeux vanu-pieds qui s'apprêtent à improviser une pièce, hors de ce cadre, les acteurs jouant sur la scène le rôle des va-nu-pieds. Dans la pièce, Oldrents (à l'image du duc Theseus dans A Midsummer Night's Dream) opte d'emblée pour l'indulgence afin d'épargner le fouet aux gueuxacteurs et déclare: "rather than they shall suffer, I will be pleas'd, let 'em play their worst" (5.1.311-12). Justice Clark finit par se laisser persuader et renonce à poursuivre et à punir les gueux-acteurs. L'année 1642 réduit l'effet de miroir entre l'illusion et la réalité à un trompe-l'œil, car si Richard Brome a la clémence et l'approbation de son mécène et de son public, il n'obtient que la vindicte des parlementaires puritains et se voit contraint de renoncer à la mise en scène de ses pièces. L'humanité que le dramaturge exprime dans les propos cléments d'Oldrents suggère qu'il étend sa défense du théâtre aux acteurs et à ceux que l'on montrait pareillement du doigt, les traîne-savates. Derrière la réécriture de la misère, l'économie du divertissement et de la contagion affective, ainsi que l'économie d'échange se devine la compréhension de celui qui, à son tour, fait l'expérience d'un statut précaire et se retrouve du côté de la marge, bien qu'il ait été apprécié de son vivant par un large auditoire. ${ }^{40}$

Pourtant, à la veille de la fermeture des théâtres, la sympathie du public pour la comédie elle-même ne fait que commencer. Rejouée dès la Restauration sous le nom de The Beggars' Chorus; Or, the Jovial Crew et rééditée plusieurs fois, elle se moule parfaitement dans un genre nouveau qui voit le jour au début du XVIIIe siècle et suscite d'emblée l'engouement des spectateurs, le "ballad-opera"; elle devient donc, en 1731, The Jovial Crew, a Comic Opera et donne lieu à des éditions musicales à part comme $A$ New Book of Song to the Jovial Crew et Airs, Songs and Duettos in the Jovial Crew. The Beggar's Opera (1728) de John Gay, probablement inspiré de A Jovial Crew, témoigne de

40. "There is a considerable amount of evidence to show that Brome was an extremely popular playwright in his day. ... The statements of many of the title-pages to the plays, Brome's own dedications and prologues, and the verses of Jonson and other friends, all agree in giving us the impression that the confidence in Brome's power to please the public ... was well justified" (Clarence E. Andrews, Richard Brome: a Study of His Life and Works (New York: Holt, 1913) 29. 
l'immense succès rencontré par ce genre innovant. ${ }^{41}$ Deux siècles plus tard exactement, Bertold Brecht reprend The Beggar's Opera pour en faire une version moderne dans L'Opéra de quat'sous. Sa pièce, le "ballad-opera" de John Gay et la comédie de Richard Brome offrent une mise en perspective diachronique qui méritait une étude en soi et qui, pour les traitements respectifs des gueux, relèverait sans doute plus de la polyphonie contrapuntique que de la résonance en sympathie.

Pascale DROUET

Université de Limoges

${ }^{41}$. Voir l'introduction de Jacques Michon dans John Gay, L'Opéra du Gueux / The Beggar's Opera (Paris: Aubier, 1983) 38. 